\title{
ÁCAROS PLANTÍCOLAS (ACARI) DA "ESTAÇÃO ECOLÓGICA DO NOROESTE PAULISTA”, ESTADO DE SÃO PAULO, BRASIL
}

\author{
Reinaldo José Fazzio Feres', Antonio Carlos Lofego ${ }^{2}$ \& Anibal Ramadan Oliveira ${ }^{2}$
}

Biota Neotropica v5 (n1) - http://www.biotaneotropica.org.br/v5n1/pt/abstract?article+BN00405012005

\author{
Recebido: 2/08/2004 \\ Revisado: $11 / 11 / 2004$ \\ Publicado: 01/01/2005
}

\begin{abstract}
${ }^{1}$ Departamento de Zoologia e Botânica, Universidade Estadual Paulista. Rua Cristóvão Colombo 2265, Jardim Nazareth, 15054-000 São José do Rio Preto, São Paulo, Brasil.

${ }^{2}$ Departamento de Zoologia, Instituto de Biociências, Universidade de São Paulo, 05508-900 São Paulo-SP, Brasil. Autor para correspondência: E-mail: reinaldo@ibilce.unesp.br
\end{abstract}

\begin{abstract}
This study was conducted in the "Estação Ecológica do Noroeste Paulista", located in the region of São José do Rio Preto, State of São Paulo, Brazil, to determine plant inhabiting mite species in patches of native forest. Eighty three mite species belonging to 48 genera in 20 families were collected on more than 60 plant species. Thirty eight mite species were nominally identified. The most frequent were Tetranychus ludeni (Zacher, 1913) (Tetranychidae) and Iphiseiodes zuluagai (Denmarkyv \& Muma, 1972) (Phytoseiidae), collected on 15 and 14 plants species, respectively. The largest diversity was observed on Piper sp. (Piperaceae) (21 mite species), Luehea speciosa Willd. (Tiliaceae (17), Alchornea glandulosa Poepp. \& Endl. (Euphorbiaceae) (16), Bauhinia rufa (Bong.) Steud (Caesalpinaceae) (14) e Olyra sp. (Poaceae)(12).
\end{abstract}

Key words: Distribution, biodiversity, mites, Neotropics, taxonomy.

\section{Resumo}

O presente estudo foi realizado na "Estação Ecológica do Noroeste Paulista", localizada na região de São José do Rio Preto, Estado de São Paulo, Brasil, visando determinar espécies acarinas plantícolas associadas a fragmentos de mata nativa. Foram determinadas 83 espécies, pertencentes a 48 gêneros de 20 famílias, associadas a mais de 60 espécies vegetais. Trinta e oito espécies acarinas foram identificadas nominalmente. As mais freqüentes foram Tetranychus ludeni (Zacher, 1913) (Tetranychidae) e Iphiseiodes zuluagai (Denmark \& Muma, 1972) (Phytoseiidae), coletadas sobre 15 e 14 espécies de plantas, respectivamente. A maior diversidade foi observada em Piper sp. (Piperaceae) (21 espécies acarinas), seguida de Luehea speciosa Willd. (Tiliaceae) (17), Alchornea glandulosa Poepp. \& Endl. (Euphorbiaceae) (16), Bauhinia rufa (Bong.) Steud (Caesalpinaceae) (14) e Olyra sp. (Poaceae) (12).

Palavras-chave: Ácaros, biodiversidade, distribuição, Região Neotropical, taxonomia. 


\section{Introdução}

Estudos sobre a biodiversidade são importantes pois, devido ao crescimento explosivo das populações humanas, o ambiente está sendo alterado de forma muito acelerada e grande parte da diversidade está se perdendo irreversivelmente através da extinção causada pela destruição de hábitats naturais (Wilson 1997). Devido à crescente devastação das matas é possível que muitas espécies de ácaros estejam sendo extintas, antes mesmo de serem catalogadas.

O conhecimento da acarofauna associada a plantas silvestres é básico para futuros estudos de manejo de agroecossistemas, pois essas plantas podem servir como reservatórios para ácaros fitófagos, além de abrigarem espécies ainda desconhecidas de inimigos naturais que poderiam se tornar disponíveis como agentes de controle biológico de pragas agrícolas.

São poucos os trabalhos enfocando a acarofauna de plantas silvestres. Feres \& Moraes (1998) registraram 15 espécies de Phytoseiidae em 18 espécies de plantas de dois fragmentos de floresta estacional semidecidual no Estado de São Paulo. Feres \& Nunes (2001) registraram 20 espécies de ácaros associadas à três espécies de euforbiáceas herbáceas que ocorrem no interior de seringais. Zacarias \& Moraes (2001) registraram 25 espécies de Phytoseiidae associadas a euforbiáceas arbóreas nativas, incluindo a seringueira, em três localidades no Estado de São Paulo. Ferla \& Moraes (2002) registraram nove espécies de Phytoseiidae em três euforbiáceas no Estado do Rio Grande do Sul. Daud \& Feres (2004) registraram 36 espécies de 15 famílias em Mabea fistulifera Mart. (Euphorbiaceae) em dois fragmentos, rural e urbano, de floresta estacional semidecidual, no Estado de São Paulo, e estudaram a diversidade, a flutuação populacional e estrutura da comunidade de ácaros associados a essa planta.

A "Estação Ecológica do Noroeste Paulista", localizada na Região de São José do Rio Preto- SP (20 50'S e $\left.49^{\circ} 26^{\prime} \mathrm{W}\right)$, se constitui em um dos últimos refúgios da fauna e flora típicas dessa região do Estado. Situada no interior do "Instituto Penal Agrícola" (IPA), entre os municípios de S. J. do Rio Preto e Mirassol, a Estação possui cerca de 170 ha relativamente bem preservados de floresta estacional semidecidual, savana arbórea, matas ciliares etc.

\section{Material e Métodos}

Com o objetivo de investigar a diversidade de ácaros em plantas silvestres dos ecossistemas que compõem a "Estação Ecológica do Noroeste Paulista", foram realizadas cinco coletas exploratórias esporádicas em 1990/1991 e 10 coletas mensais em 1992. Foram amostradas partes aéreas das plantas (principalmente folhas) que apresentavam sintomas de infestação por ácaros fitófagos ou que tiveram sua presença detectada com auxílio de uma lupa de bolso (10-15x), durante as coletas. Foram tomadas amostras de mais de 60 espécies de plantas, tendo sido possível a identificação de 23 delas. As amostras foram embaladas em sacos de polietileno e acondicionadas em caixas isotérmicas de poliestireno com bolsas de gelo no interior. No laboratório, os ácaros foram extraídos sob estereoscópio, montados em lâminas de microscopia com meio de Hoyer (Flechtmann 1975) e identificados sob microscópio óptico com contraste de fase.

A nomenclatura adotada para as categorias superiores foi aquela proposta por Woolley (1988). A distribuição mundial das espécies de Tetranychidae e Phytoseiidae foi baseada em Bolland et al. (1998) e Moraes et al. (2004), respectivamente, e conforme indicado no texto para as espécies dos demais grupos. $\mathrm{Na}$ apresentação dos resultados, precedendo o nome da espécie vegetal coletada no item "material examinado", os algarismos arábicos nos primeiros dígitos indicam o número de exemplares determinados e nos dois últimos dígitos os anos em que foram realizadas as coletas. Os algarismos romanos se referem aos meses das coletas. O sexo e a fase de desenvolvimento dos espécimes são indicados através das seguintes abreviaturas: (f) fêmea, (m) macho, (n) ninfa, (l) larva.

Os espécimes estudados foram depositados na coleção de Acari (DZSJRP) do Departamento de Zoologia e Botânica, Universidade Estadual Paulista (UNESP), São José do Rio Preto, São Paulo.

\section{Resultados}

O estudo taxonômico dos exemplares revelou a ocorrência de 83 espécies de ácaros (38 identificadas nominalmente) pertencentes a 48 gêneros de 20 famílias.

\section{Gamasida}

Ascidae Voigts \& Oudemans, 1905

Asca sp.

Material examinado. 1m IV-92, 1fVI-92, 1fVII-92, 1f X-92, Acalypha sp. (Euphorbiaceae); if VII-92, Alchornea glandulosa Poepp. \& Endl. (Euphorbiaceae); $1 \mathrm{f}$ VI-92, Bauhinia rufa (Bong.) Steud. (Caesalpinaceae); $1 \mathrm{f}$ IV-92, Desmodium canum (J.F.Gmel.) Schinz et Thell (Fabaceae); 2f IV-92; 1n II-92, 1m IV-92, Lantana sp. (Verbenaceae); 3f e 1m III-92, 2f e 1m IV-92, 1fVI-92, Luehea speciosa Willd. (Tiliaceae); 2f I-91, 1fIV-91, 1f III-92, $2 \mathrm{fe} 1 \mathrm{~m}$ IV-92, 2f , 1m e 1n VI-92, 1fX-92, plantas não identificadas.

Phytoseiidae Berlese, 1913

Amblyseius acalyphus Denmark \& Muma, 1973 Amblyseius acalyphus Denmark \& Muma, 1973: 242, 1989 : 75; Moraes et al., 1986: 6; Feres \& Moraes, 1998: 125. 
Material examinado. 1f VI-92, 1fVII-92, L. speciosa; 1f VI-92, 1f VII-92, Styrax camporum Pohl(Styracaceae). Distribuição. Brasil- São Paulo

\section{Amblyseius aerialis (Muma, 1955)}

Amblyseiopsis aerialis Muma, 1955: 264; Garman, 1958: 75. Typhlodromus (Amblyseius) aerialis, Chant, 1959: 88. Amblyseius aerialis, Athias-Henriot, 1957: 338; Muma, 1961: 287; De Leon, 1966: 91; Moraes et al., 1986: 6; Moraes \& Mesa, 1988: 71; Denmark \& Muma, 1989: 15; Kreiter \& Moraes, 1997: 377; Feres \& Moraes, 1998: 126; Moraes et al., 2000: 238; Gondim Jr. \& Moraes, 2001: 67. (Piperaceae).

Material examinado. 1f VI-92, Piper sp.

Distribuição. Argélia; Bermudas; Brasil- Bahia, Pernambuco, São Paulo; Colômbia; Cuba; EUA; Galápagos; Guadalupe; Guiana; Honduras; Índia; Jamaica; Martinica; México; Venezuela.

\section{Amblyseius chiapensis De Leon, 1961b}

Amblyseius chiapensis De Leon, 1961b: 85; De Leon, 1962: 175; McMurtry, 1983: 250; Moraes \& Mesa, 1988: 72; Moraes et al., 1986: 10; Denmark \& Muma, 1989: 94; McMurtry \& Moraes, 1989: 185; Gondim Jr. \& Moraes, 2001: 67.

Amblyseius triplaris De Leon, 1967: 25 (sinônimo júnior, de acordo com Denmark \& Muma, 1989: 94).

Material examinado. 1f VI-92, 1m VII-92, $S$. camporum; 1f XII-92, Piper sp.; 1f VII-92, 1f-X-92, 1f XII92, três plantas não identificadas.

Distribuição. Brasil- Bahia, Ceará; Maranhão, Pernambuco, Rio Grande do Sul, Santa Catarina, São Paulo; Colômbia; El Salvador; Guatemala; Honduras; México; Porto Rico; Venezuela.

Amblyseius compositus Denmark \& Muma, 1973

Amblyseius compositus Denmark \& Muma, 1973: 240, 1989: 95; Moraes et al., 1986: 11; Gondim Jr. \& Moraes, 2001 : 67.

Material examinado. 1f e 1m XII-92, A. glandulosa; 2f IX-92, Celtis iguanae (Jacq.) Sarg. (Ulmaceae); 3f VI-92, L. speciosa; 1f VII-92, Olyra sp. (Poaceae); $1 \mathrm{~m}$ VI-92, 1fIX92, 1f XII-92, Piper sp.

Distribuição. Brasil- Bahia, Piauí, São Paulo.

\section{Euseius concordis (Chant, 1959)}

Typhlodromus (Amblyseius) concordis Chant, 1959: 69.

Amblyseius (Iphiseius) concordis, Muma, 1961: 288.

Amblyseius concordis, Chant \& Baker, 1965: 22

Euseius concordis, Denmark \& Muma, 1973: 264; Moraes \& Oliveira, 1982: 317; Moraes \& McMurtry, 1983: 138; Moraes et al., 1986: 39; Feres \& Moraes, 1998: 127; Gondim Jr. \& Moraes, 2001: 74.

Euseius flechtmanni, Denmark \& Muma, 1970: 223, 1973: 261 (sinônimo júnior, de acordo com Moraes et al., 1982: 18)
Material examinado. 2f IIII-92, $1 \mathrm{fVII}-92$, $1 \mathrm{~m}$ XII-92, Pipersp.; 6f III-92, 1fIV-92, 1fVI-92, 1fe 1m VII-92, 6f e 1m IX-92, 1f X-92, três plantas não identificadas.

Distribuição. Argentina; Brasil- Bahia, Ceará, Minas Gerais, Paraíba, Pernambuco, Piauí, Rio Grande do Sul, São Paulo; Colômbia; Costa Rica; El Salvador; Guatemala; Honduras; Monte Negro; Nicarágua; Paraguai; Portugal; Trinidad e Tobago; Venezuela.

\section{Iphiseiodes zuluagai Denmark \& Muma, 1972}

Iphiseiodes zuluagai Denmark \& Muma, 1972: 23; Denmark \& Muma, 1973: 251; Denmark \& Muma, 1975: 287; Moraes et al., 1982: 18; Moraes et al., 1986: 61; Aponte \& McMurtry, 1995: 176; Kreiter \& Moraes, 1997: 377; Feres \& Moraes, 1998: 127; Moraes et al., 1999 (2000): 245; Gondim Jr. \& Moraes, 2001: 76.

Amblyseius zuluagai, Moraes \& Mesa, 1988: 79; Moraes et al., 1991: 125 .

Material examinado. 2fIII-92, 2fIV-92, 1fVII-92, $A$. glandulosa; 1m VII-92, B. rufa; 2f VII-92, C. iguanae; 1f VIII-91, 1m IV-92, 1fIX-92, L. speciosa; 2f e $1 \mathrm{~m}$ IV-92, 1fVI92, 1f VII-92, 3f e 1m IX-92, 1f X-92, Piper sp.; 3f VI-92, Psidium guajava L. (Myrtaceae); 2f X-92, Asteraceae não identificada; 1m IV-92, 2fVII-92, 6f e 1m IX-92, oito plantas não identificadas.

Distribuição. Brasil- Bahia, Maranhão, Minas Gerais, Pernambuco, Rio Grande do Sul, São Paulo; Colômbia; Cuba; Guadalupe; Marie Galante; Martinica; Panamá; Porto Rico; Venezuela.

\section{Neoseiulus bellottii (Moraes \& Mesa, 1988)}

Amblyseius bellottii Moraes \& Mesa, 1988: 75.

Neoseiulus bellottii; Moraes et al., 2004: 108.

Material examinado. $1 \mathrm{fe} 1 \mathrm{~m}$ III-92, Helicteres sp. (Sterculiaceae); 1fe 1m III-92, 2f e 1m IV-92, 3fVI-92, 1fIX92, L. speciosa; 1m IV-92, Piper sp.; 3f III-92, 1fVI-92, planta não identificada.

Distribuição. Brasil- Bahia; Colômbia.

\section{Neoseiulus tunus (De Leon, 1967)}

Typhlodromips tunus De Leon, 1967: 29; Denmark \& Muma, 1973: 253; Moraes et al., 1986: 151; Feres \& Moraes, 1998: 126.

Amblyseius tunus, McMurtry \& Moraes, 1989: 181.

Material examinado. 1f III-92, L. speciosa; 2f VI92, 4f VII-92, 1f XII-92, Piper sp.; 1f IX-92, Olyra sp.; 1fX92, Lippia sp.; 2f III-92, 1f VI-92, 1f X-92, duas plantas não identificadas.

Distribuição. Brasil- Rio Grande do Sul, São Paulo; Guadalupe; Jamaica; Marie Galante; Martinica; Peru; Trinidad e Tobago.

Phytoseiulus macropilis (Banks, 1905)

Laelaps macropilis Banks, 1905: 139. 
Hypoaspis macropilis, Banks, 1915: 85.

Phytoseiulus speyeri Evans, 1952: 398 (sinônimo júnior, de acordo com Kennett, 1958: 477).

Phytoseiulus chanti Ehara, 1966: 135 (sinônimo júnior, de acordo com Denmark \& Muma, 1973: 236).

Phytoseiulus macropilis, Cunliffe \& Baker, 1953: 23; Denmark \& Muma, 1970: 30; McMurtry, 1983: 259; Denmark \& Schicha, 1983: 31; Moraes et al., 1986: 108; Kreiter \& Moraes, 1997: 378; Gondim Jr. \& Moraes, 2001: 81 .

Material examinado. $1 \mathrm{~m}$ VI-90, Centrosema pubescens Benth (Fabaceae); 1m X-92, Lantana lilacina Desf. (Verbenaceae), 1fIX-92, Piper sp.; 2f 26-IV-91, 1m IX92, duas plantas não identificadas.

Distribuição. Angola; Argentina; Barbados; BrasilCeará, Minas Gerais, Rio Grande do Sul, São Paulo; Canadá; Colômbia; Costa Rica; Cuba; E.U.A.; Fiji; Guadalupe; Guatemala; Havaí; Honduras; Ilhas Canárias; Ilhas Cook; Jamaica; Les Saintes; Marie Galante; Martinica; México; Monte Negro; Moto Quavarei; Nova Caledônia; Panamá; Peru; Polinésia Francesa; Polônia, Porto Rico; Portugal; San Martin; Tahiti; Venezuela.

\section{Phytoseiulus fragariae Denmark \& Schicha, 1983}

Phytoseiulus fragariae Denmark \& Schicha, 1983: 34;

Moraes et al., 1986: 108; Moraes et al., 1991: 132. identificada.

Material examinado. If IX-92, planta não Colômbia.

Distribuição. Argentina; Brasil- São Paulo;

Phytoseius guianensis De Leon, 1965

Phytoseius (Pennaseius) guianensis De Leon, 1965: 15.

Phytoseius guianensis, Denmark \& Muma, 1973: 269;

Moraes \& McMurtry, 1983: 144; Moraes et al., 1986:

211; Gondim jr. \& Moraes, 2001: 87.

Material examinado. 1f VII-92, B. rufa; $1 \mathrm{fIII}-92,3 \mathrm{f}$

IV-92, 2fVI-92, 1fVII-92, 5f IX-92, planta não identificada.

Distribuição. Brasil- Bahia, Ceará, Paraíba,

Pernambuco, Piauí, Rio Grande do Sul; São Paulo; Guiana; Venezuela.

Phytoseius nahuatlensis De Leon, 1959

Phytoseius nahuatlensis De Leon, 1959: 147; Chant \& Baker, 1965: 56.

Phytoseius (Phytoseius) nahuatlensis, Chant, 1959: 106; Denmark, 1966: 25 .

Phytoseius (Pennaseius) nahuatlensis, De Leon, 1965: 14;

Moraes et al., 1986: 213.

Material examinado. 9f VIII-91, planta não identificada.

Distribuição. Brasil- São Paulo; Costa Rica; México; Nicarágua.
Proprioseiopsis neotropicus (Ehara, 1966)

Amblyseius neotropicus Ehara, 1966: 133; Moraes \& Mesa, 1988: 79; Moraes et al., 1991: 126.

Proprioseiopsis neotropicus, Moraes et al., 1986: 119; Gondim \& Moraes, 2001: 81.

Material examinado. 1m XI-92, A. glandulosa; $1 \mathrm{f}$ VI-92, 1f VII-92, B. rufa; $1 \mathrm{fe} 1 \mathrm{~m}$ VIII-92, L. speciosa; 1f III92, 3f e 1m VI-92, Piper sp.; 1f III-92, 1m X-92, duas plantas não identificadas.

Distribuição. Brasil- Pernambuco, Rio Grande do

Sul, São Paulo; Colômbia; Equador.

Galendromus (Galendromus) annectens De Leon, 1958

Typhlodromus annectens De Leon, 1958: 75; Chant \& Yoshida-Saul, 1984: 1868; Moraes \& McMurtry, 1983: 142; Moraes \& Mesa, 1988: 82; Moraes et al., 1991: 134; Feres \& Moraes, 1998: 128; Feres, 2000: 161; Feres \& Nunes, 2001: 1256.

Galendromus annectens, Muma, 1961: 298; Muma, 1963: 20; Muma et al. 1970: 135; Denmark \& Muma, 1973: 274; Farias et al., 1981:21; Denmark, 1982: 142; Moraes et al., 1982: 21; Moraes et al., 1986: 186; Gondim Jr. \& Moraes, 2001: 88.

Material examinado. 3f IX-92, duas plantas não identificadas.

Distribuição. Brasil- Bahia, Ceará, Minas Gerais, Pernambuco, Rio Grande do Sul, São Paulo; Canadá; Colômbia; Costa Rica; Cuba; El Salvador; E.U.A.; Galápagos; Honduras; Jamaica; México; Porto Rico; Venezuela.

Typhlodromalus aripo De Leon, 1967

Typhlodromalus aripo De Leon, 1967: 21; Denmark \& Muma, 1973: 257; Moraes et al., 1986: 128; Moraes et al., 1999 (2000): 252.

Amblyseius aripo, Moraes \& McMurtry, 1983: 132; Moraes \& Mesa, 1988: 73; Feres \& Moraes, 1998: 126.

Material examinado. If X-92, Asteraceae não identificada.

Distribuição. Brasil- Bahia, Ceará, Maranhão, Pernambuco, Piauí, Rio Grande do Sul, São Paulo; Colômbia; Costa Rica; El Salvador; Guadalupe; Guiana; Jamaica; Paraguai; Trinidad e Tobago.

\section{Typhlodromalus sp.}

Material examinado. If VI-92, S. camporum; if VII-92, Stryphnodendron adstringens (Mart.) Coville (Mimosaceae); 1f VIII-91, 2f VII-92, duas plantas não identificadas.

"Incertae sedis"

Africoseius sp. 

identificada

Material examinado. 1f IV-91, planta não

Actinedida

\section{Eupodidae Koch, 1842}

\section{Eupodes sp. (cf. parafusifer)}

Material examinado. If IX-92, Olyra latifolia L. (Poaceae); 1f VIII-90, planta não identificada.

\section{Cheyletidae Leach, 1815}

Cheletomimus (Hemicheyletia) wellsi (Baker, 1949a) Cheyletia wellsi Baker, 1949a: 300-301.

Paracheyletia wellsi, Volgin, 1955: 152; Muma, 1964: 245 246.

Dendrocheyla wellsi, Volgin, 1969: 211.

Hemicheyletia wellsi, Summers \& Price, 1970: 18.

Cheletomimus (Hemicheyletia) wellsi, Fain, Bochkov \& Corpuz-Rarus, 2002: 45. identificada.

Material examinado. 1f VII-92; 1f IX-92, planta não

Distribuição. África (Rodrigues 1968); Brasil- São Paulo (Feres \& Flechtmann 1995); E.U.A. (Muma 1964).

Observações. A maioria das espécies é predadora de grande variedade de pequenos artrópodes, como ácaros e colêmbolos. Algumas espécies ocorrem sobre a folhagem das plantas, onde se alimentam de ácaros fitófagos e cochonilhas (Flechtmann 1975). Hemicheyletia wellsi tem sido registrada com freqüência em folhas e frutos de citros atacados por Phyllocoptruta oleivora (Ashmead) (Eriophyidae), o ácaro da falsa ferrugem do citros (Chiavegato 1980).

Cunaxidae Thor, 1902

Cunaxoides sp.

Material examinado. 1f IV-92, B. rufa; 2f VII-92, planta não identificada.

Observações. Ácaros da família Cunaxidae são predadores de cochonilhas e outros pequenos artrópodes (Smiley 1975).

\section{Stigmaeidae Oudemans, 1931}

\section{Agistemus sp.1}

Material examinado. 1fVI-92, Acalypha sp.; 1fVII92, A. glandulosa; 1n e 11 VIII-90, 2f e 1m VIII-91, Bauhinia sp.; 1F, 1m e 1n III-92, B. rufa; 2f III-92, 1f IV-92, Lantana sp.; 3m IX-92, 1m X-92, L. lilacina; 1n III-92, 4f e 2n IV-92, 1f e 2n VII-92, $1 \mathrm{~m}$ e 1n IX-92, L. speciosa; 1f VI-92, Olyra sp.; 1fIX-92, Piper sp.; 1f VII-92, S. adstringens; 1f VIII-90, 1f, 1m e 1n VIII-91, 1m IV-92, 1f e 1m X-92, 1f e 1m XII-92, plantas não identificadas.

\section{Agistemus sp.2}

Material examinado. 2f IV-92, A. glandulosa; 1f, $1 \mathrm{~m}$ e 3n IV-92, 1f X-92, 2f XII-92, B. rufa; 1f IX-92, Lippia sp.; 1f e 2n VI-92, L. speciosa; 1f e 1n III-92, 2f e 3m IX-92, plantas não identificadas.

Observações. Espécies de Agistemus são geralmente conhecidas como predadoras ativas de ovos de tetraniquídeos (McMurtry et al. 1970; Oomen 1982 e Inoue \& Tanaka 1983 apud Ehara 1993). São ácaros referidos como predadores de Tenuipalpus spp. (Flechtmann 1975). Muito comuns, ocorrendo sobre grande número de plantas. Sua coloração varia do brancacento ao vermelho amarronzado.

\section{Zetzellia sp.}

Material examinado. 1n IX-92, Lippia sp.; 1n IX92, Piper sp.; $1 \mathrm{~m}$ XI-92, planta não identificada.

Distribuição. Gênero com espécies de ampla distribuição geográfica (Wood 1967).

Observações. É conhecido o hábito alimentar de Zetzellia mali (Ewing), que se alimenta de várias espécies de ácaros tetraniquídeos na América do Norte, Europa e Israel (Jeppson et al. 1975).

\section{Ledermuelleria sp.}

Material examinado. 2m VI-92, S. adstringens.

$$
\text { Tydeidae Kramer, } 1877
$$

\section{Lorryia formosa Cooreman, 1958}

Lorryia formosa Cooreman,1958: 6-10; Baker, 1968: 995-996.

Material examinado. 1f VI-92, 2f e 2n VII-92, 1fXII92, A. glandulosa; 1n XII-92, Olyra sp.; 4f IX-92, Piper sp.; 2fe 5n 25-IV-91, 14f IX-92, 2n X-92, plantas não identificadas.

Distribuição. Argentina; Brasil; Equador; Espanha; França; Marrocos; México; Uruguai (Baker 1968); Paraguai (Aranda \& Flechtmann 1969, Flechtmann 1973).

Observações. Ácaros de coloração verdeamarelada. Uma espécie não identificada deste gênero foi registrada em seringueiras do Município de Rio Claro, SP, por Flechtmann \& Arleu (1984). Ocorrem praticamente ao longo de todo o ano, sobre um grande número de plantas, sem causar dano aparente às folhas.

\section{Lorryia sp.}

Material examinado. 1f IX-92, Acalypha sp.

\section{Tydeus sp.}

Material examinado. 2f III-92, $1 \mathrm{fIV}-92,1 \mathrm{fXI}-92 \mathrm{~A}$. glandulosa; 1f IV-92 B. rufa; 1f IX-92 Lippia sp.; 1 IX IX-92 Olyra sp.; 1f e 1n III-92, Piper sp.; 1n VI-92 Stachytarphetta 
cayenensis (L.C. Rinch) Vahl (Verbenaceae); 1n III-92, $1 \mathrm{f}$ IV-92, 3n IX-92, plantas não identificadas.

Observações. Pouco se conhece a respeito da biologia das espécies pertencentes a este gênero. Podem ser encontrados em grande número, sobre folhas de plantas diversas, no húmus, fragmentos vegetais e sobre produtos alimentícios armazenados (Baker 1970).

\section{Pronematus sp.}

Material examinado. 1f XII-92, Acalypha sp.; 1f III-92, A. glandulosa; 4f IX-92, 1m 21-X-92, Bambusa sp. (Poaceae); 1fIV-92, 1fVI-92, B. rufa; 1fe 1n X-92, L. lilacina; 1f IX-92, Olyra sp.; 2f e 2m X-92, planta não identificada.

Observações. Ácaros diminutos, translúcidos, levemente brancacentos e de movimentos rápidos. São referidos como predadores de eriofiídeos (Baker \& Wharton 1952).

\section{Camerobiidae Southcott, 1957}

\section{Neophyllobius sp.}

Material examinado. 1f III-92, L. speciosa.

$$
\text { Eupalopsellidae Willman, } 1952
$$

\section{Exothorhis sp.}

Material examinado. 1f VI-92, 1fVII-92, planta não identificada.

\section{Tarsonemidae Canestrini \& Fanzago, 1877}

\section{Daidalotarsonemus tesselatus De Leon, 1956}

Daidalotarsonemus tesselatus De Leon, 1956: 163; Smiley, 1972: 91.

Material examinado. 1m III-92, A. glandulosa; $1 \mathrm{f}$ VI-92, B. rufa; 1f VI-92, P. guajava; 1f VI-92, 1f VII-92, S. camporum.

Distribuição. E.U.A e Japão (Lin \& Zhang 2002).

Observações. De Leon (1956) sugere que esta espécie seja fitófaga em Litchi Sonn. (Sapindaceae). Suski (1967), entretanto, não exclui a possibilidade de que se alimente também de líquens e fungos, como provavelmente o fazem as demais espécies do gênero (Lindquist 1986).

Daidalotarsonemus sp.

Material examinado. 1f VI-92, S. santaremensis.

\section{Metatarsonemus sp.}

Material examinado. 1fIX-92, 1fX-92, duas plantas não identificadas.

Distribuição. E.U.A. (Attiah 1970).

Observações. Não há estudos sobre os hábitos alimentares de ácaros deste gênero, no entanto, a coloração do conteúdo intestinal, verde clara nas larvas e verde escura nos adultos, pode ser um indicativo de que sejam fitófagos.

\section{Tarsonemus bilobatus Suski, 1965}

Tarsonemus bilobatus Suski, 1965: 539

Material examinado. 1f VI-92, A. glandulosa; if IV-92, D. canum.

Distribuição. Bielorussia, China, Coréia, Costa Rica, Egito, Hungria, Itália, Japão, Polônia e Ucrânia (Lin \& Zhang 2002).

Observações. Ácaros deste gênero são primariamente micófagos (Lindquist 1986).

\section{Tarsonemus confusus Ewing, 1939}

Tarsonemus confusus Ewing, 1939: 26; Smiley 1969: 221; Kaliszewski, 1993: 40.

Material examinado. 1f 09-92, L. lilacina.

Distribuição. Alemanha, Bielorussia, China, Coréia, E.U.A., Holanda, Irlanda, Itália, Japão, Polônia, Turquia e Ucrânia (Lin \& Zhang 2002).

\section{Tarsonemus waitei Banks, 1912}

Tarsonemus waitei Banks, 1912: 96; Lindquist, 1978: 1024. Tarsonemus setifer Ewing, 1939: 19 (sinônimo júnior de acordo com Lindquist, 1978).

Tarsonemus pauperoseatus Suski, 1967: 267 (sinônimo júnior de acordo com Lindquist, 1978).

Material examinado. 1fX-92, B. rufa.

Distribuição. Brasil, Canadá, China, Congo, Coréia, Costa Rica, Egito, E.U.A., Nova Zelândia, Polônia, Portugal, Turquia e Ucrânia (Lin \& Zhang 2002).

Observações. Pode ser fitófaga facultativa ou transicional, ocasionalmente em hospedeiros de importância agrícola, como relatado por Lindquist (1978) e Karl (1965) apud Lindquist (1986).

\section{Tarsonemus sp.1}

Material examinado. 1fVI-92, D. canum; 1F, 7M, 2L, IV-92, S. santaremensis; 1f VII-92, Z. stipitatum.

\section{Tarsonemus sp.2}

Material examinado. 1f III-92, Lantana sp.

\section{Xenotarsonemus sp.1}

Material examinado. 3f VI-92, Lantana sp.; 2f III92, 2f VI-92, 1f IX-92, L. speciosa; 1f VII-92, Olyra sp.; 1f e 1m IX-1992, T. semitriloba; 1f VII-92, Vignia sp.; 1f VI-92, 3f VIII-91, 5f e 1m IX-92, 1f X-92, quatro plantas não identificadas.

Distribuição. Espécies do gênero ocorrem em regiões temperadas e tropicais, com ampla distribuição geográfica (Lindquist 1986).

Observações. Hábito alimentar não definido (Lindquist 1986). 


\section{Xenotarsonemus sp.2 \\ Material examinado. 3f VII-92, Acalypha sp.}

Xenotarsonemus sp.3

Material examinado. 1fIV-92, D. canum; 1f VII-92, Vignia sp.; 1f IV-92, planta não identificada.

\section{Xenotarsonemus sp.4} identificada.

Material examinado. 1f X-92, planta não

\section{Xenotarsonemus sp.5 \\ Material examinado. 1fVII-92, Vignia sp.}

Tenuipalpidae Berlese, 1913

\section{Brevipalpus californicus (Banks, 1904)}

Tenuipalpus californicus Banks, 1904: 55.

Brevipalpus californicus, Pritchard \& Baker, 1958: 216; De Leon, 1961a: 46; Baker, Tuttle \& Abbatiello, 1975: 16; Meyer, 1979: 89; Baker \& Tuttle, 1987: 32.

Material examinado. 1f VII-92, Piper sp.

Distribuição. Espécie de ampla distribuição geográfica, ocorrendo sobre grande número de espécies de plantas hospedeiras. África- África do Sul, Angola, Líbia, Moçambique, Nigéria, Rodésia, Zaire, Maurício, Egito, Malaui, Uganda (Meyer 1979); Brasil- Bahia, São Paulo; México (Baker \& Tuttle 1987).

\section{Brevipalpus obovatus Donnadieu, 1875}

Brevipalpus obovatus Donnadieu, 1875: 116; Pritchard \& Baker, 1958: 231; De Leon, 1961a: 48; Baker, Tuttle \& Abbatiello, 1975: 18; Meyer, 1979: 86; Baker \& Tuttle, 1987: 96.

Brevipalpus pereger, Donnadieu, 1875: 116.

Tenuipalpus inornatus, Banks, 1912: 97.

Tenuipalpus bioculatus, McGregor, 1914: 354.

Tenuipalpus pseudocuneatus, Blanchard, 1940: 11.

Brevipalpus inornatus, Pritchard \& Baker, (1951) 1952: 36.

Material examinado. 6f IV-92,3fVI-92, L. speciosa; 2fIII-92, 2f e 1n IV-92, 3fVI-92, 1fVII-92, 20f e 10n IX-92, 1f X-92, 2f e 1n XII-92, plantas não identificadas.

Distribuição. Espécie de ampla distribuição geográfica, ocorrendo sobre grande número de espécies de plantas hospedeiras. África- Angola, Egito, África do Sul, Moçambique, Malaui, Líbia, Rodésia, Uganda, Quênia (Meyer 1979); Brasil- Bahia, São Paulo (Flechtmann 1976a); México (Baker \& Tuttle 1987).

Brevipalpus phoenicis (Geijskes, 1939)

Tenuipalpus phoenicis Geijskes, 1939: 23.

Brevipalpus phoenicis, Sayed, 1946: 99; Pritchard \& Baker, 1958: 233; De Leon, 1961a: 48; Gonzalez, 1975: 82; Baker, Tuttle \& Abbatiello, 1975: 18; Meyer, 1979: 87; Baker \& Tuttle, 1987: 98-99.
Brevipalpus yothersi, Baker, 1949b: 374.

Brevipalpus mcbridei, Baker, 1949b: 374.

Brevipalpus papayensis, Baker, 1949b: 379.

Material examinado. 7f e 1n III-92, 2f IV-92, 1f VII-

92, Lantana sp.; 1f XII-92, Lippia sp; 1f IV-92, Sida santaremensis Monteiro (Malvaceae); $5 \mathrm{f} \mathrm{X-92,S.}$ cayenensis; 6f e 1n VII-92, S. adstringens; 1f VII-92, Triumfetta semitriloba Jacq. (Tiliaceae); $7 \mathrm{f}$ e $1 \mathrm{n} 28-\mathrm{I}-91,4 \mathrm{f}$ VIII-91, 1f III-92, 2f IV-92, 3f VI-92, 12f e 2n IX-92, 4f X-92, plantas não identificadas.

Distribuição. Espécie de ampla distribuição geográfica, ocorrendo sobre grande número de espécies de plantas hospedeiras. África- África do Sul, Moçambique, Egito, Rodésia, Malaui, Angola, Nigéria, Maurício, Sudão, Uganda, Quênia, Tanzânia (Meyer \& Rodrigues 1965; Rodrigues 1968; Meyer 1979); Brasil-Alagoas, Bahia, Ceará, Minas Gerais, Paraná, Pernambuco, Rio de Janeiro, São Paulo (Flechtmann 1976a); Colômbia (Zuluaga \& Saldarriaga 1970); E.U.A. (Baker \& Tuttle 1987); Porto Rico (Comroy 1958).

Observações. Referido como "ácaro da leprose dos citros", pois é o vetor da virose que causa essa patogenia nos citros (Chiavegato 1980). Espécie registrada em seringueiras de Itabuna, BA e Rio Claro, SP (Flechtmann \& Arleu 1984)

\section{Tetranychidae Donnadieu, 1875}

Allonychus reisi Paschoal, 1970
Allonychus reisi Paschoal, 1970: 84 apud Flechtmann, 1976b: 115.

Material examinado. 10f e 2m IV-91, 6f e 2m VIII-91, três plantas não identificadas.

Distribuição. Brasil, Colômbia.

\section{Aponychus sp. (cf. schultzi)}

Material examinado. 10f e 5m VII-90, $C$. pubescens ; 2f VII-92, 1f X-92, 1f XII-92, Acalypha sp.

Observações. Os exemplares coletados em Acalypha sp. apresentam as setas do par $v_{2}$ maiores, 94 (87-103 $\mu \mathrm{m})$, em relação às dos espécimes coletados em $C$. pubescens, 51 (43-54 $\mu \mathrm{m})$.

\section{Atrichoproctus uncinatus Flechtmann, 1967}

Atrichoproctus uncinatus Flechtmann, 1967: 39 apud Flechtmann \& Baker, 1970: 157; Flechtmann \& Baker, 1975: 116; Feres, 2000: 166.

Material examinado. 2f VII-92, Bauhinia sp.; $1 \mathrm{f}$ XII-92, B. rufa; 1n VII-92, S. adstringens.

Distribuição. Brasil; Colômbia; Cuba.

\section{Eotetranychus tremae De Leon, 1957}

Eotetranychus tremae De Leon, 1957: 111; Baker \& Pritchard, 1962: 319; Flechtmann, 1996: 211; Flechtmann, 2004: 10. 
Material examinado. 1fe 2m IX-92, $1 \mathrm{fe} 1 \mathrm{~m}$ XII-92, Acalypha sp.; 4f e 2m IX-92, C. iguanae; 2f, 3m e 2n III-92, 14f e $5 \mathrm{~m}$ VII-92, 8f e 1m IX-92, Piper sp.; 1fe 1m X-92, Sida sp.; 3f e 5m IX-92, 1f e 1n X-92, 4F, 2m e 11, XII-92, plantas não identificadas.

Distribuição. Brasil; Colômbia; Equador; E.U.A.; Honduras; México; Nicarágua.

\section{Eotetranychus sp.1}

Material examinado. 2fe $1 \mathrm{~m}$ VII-90, 8fe $7 \mathrm{~m}$ VIII-90, $11 \mathrm{fe} 3 \mathrm{~m}$ VIII-90, 9f VIII-91, Bauhinia sp.

\section{Eotetranychus sp.2}

Material examinado. if e $2 \mathrm{~m}$ IV-92, $S$. santaremensis.

\section{Eotetranychus sp. (aff. mastichi)}

Material examinado. $6 \mathrm{f}$ e 2m X-92, Sida sp.

Mononychellus mcgregori (Flechtmann \& Baker, 1970) Eotetranychus planki (McGregor) (parte); Pritchard \& Baker, 1955: 150.

Mononychus mcgregori Flechtmann \& Baker, 1970: 160.

Mononychellus mcgregori, Wainstein, 1971: 589; Flechtmann \& Baker, 1975: 117 identificada.

Material examinado. 2f e $1 \mathrm{~m}$ XII-92, planta não

Distribuição. Argentina; Brasil; Colômbia; Trinidad; Venezuela.

\section{Mononychellus planki (McGregor, 1950)}

Tetranychus planki Mcgregor, 1950: 300.

Eotetranychus planki Pritchard \& Baker, 1955: 148

Mononychus planki Wainstein, 1960: 198; Tuttle \& Baker, 1968: 105.

Schizotetranychus planki Livshits \& Salinas Cr., 1968: 56 Mononychellus planki, Wainstein, 1971: 589.

Mononychellus waltheria Tuttle, Baker \& Abbatiello, 1974: 9 (sinônimo, de acordo com Tuttle, Baker \& Abbatiello, 1976:59).

Material examinado. 2f e 1n III-92, 4f e 4m IV-92, Desmodium purpureum (Mill.) Fawc. et Rendle; 1f XII-92, Sida cordifolia L. (Malvaceae); 1f IV-92, 1n VII-92, S. santaremensis.

Distribuição. Argentina; Brasil; Colômbia; Costa Rica; Cuba; El Salvador; Equador; E.U.A.; Honduras; México; Paraguai; Porto Rico; Trinidad; Venezuela.

Neotetranychus granifer Feres \& Flechtmann, 2000 Neotetranychus granifer Feres \& Flechtmann, 2000: 220. Material examinado. 2f, $1 \mathrm{~m}$ e 1n III-92, 2f e 1n IV, 3F, 2m e 1n VII, 1m XII-92, B. rufa.

Distribuição. Brasil (Feres \& Flechtmann 2000).
Neotetranychus asper Feres \& Flechtmann, 2000

Neotetranychus asper Feres \& Flechtmann, 2000: 224; Flechtmann, 2004: 10.

Material examinado. 2n III-92, 1f IV-92, 2f e 1n VI92, 2f e 2m VII-92, A. glandulosa.

Distribuição. Brasil (Feres \& Flechtmann 2000).

Oligonychus gossypii (Zacher, 1921)

Paratetranychus gossypii Zacher, 1921: 183.

Oligonychus gossypii, Pritchard \& Baker, 1955: 359; Baker \& Pritchard, 1960: 508; Meyer, 1974: 263; Meyer, 1987: 152; Feres, 2000: 166.

Material examinado. 3f, 1m e 2n IV-92, 1n VI-92, planta não identificada.

Distribuição. Angola; Benin; Brasil; Camarões; República da África Central; Colômbia; Congo; Costa Rica; Equador; Etiópia; Honduras; Madagascar; Nigéria; Quênia; São Tomé; Senegal; Serra Leoa; Tanzânia; Togo; Uganda; Venezuela; Zaire.

Observações. Registrada em várias espécies de plantas. Habitam a superfície inferior das folhas, onde produzem teias.

\section{Oligonychus punicae (Hirst, 1926)}

Paratetranychus punicae Hirst, 1926: 830.

Oligonychus punicae, Pritchard \& Baker, 1955: 335.

Material examinado. 2f e 2m VII-92, 2f e 3m IX-92, Olyra sp.

Distribuição. Austrália; Brasil; Chile; China; Colômbia; Costa Rica; Cuba; Egito; El Salvador; E.U.A.; França; Guatemala; Honduras; India; México; Nicarágua; Panamá e Venezuela.

Oligonychus yothersi (McGregor, 1914)

Tetranychus yothersi McGregor, 1914: 355.

Oligonychus yothersi, Pritchard \& Baker, 1955: 330; Baker \& Pritchard, 1962: 322; Flechtmann \& Baker, 1970: 156. identificada.

Material examinado. 4f e 3m IX-92, planta não

Distribuição. Argentina; Brasil; Chile; China; Colômbia; Costa Rica; Cuba; Equador; E.U.A.; Havaí; México; Nicarágua; Paraguai; Peru.

\section{Oligonychus sp.}

Material examinado. 16f e 3n VIII-90, planta não identificada.

\section{Oligonychus zeae (McGregor, 1955)}

Paratetranychus zeae McGregor, 1955: 370.

Oligonychus zeae, Baker \& Pritchard, 1962: 328; Flechtmann \& Santana, 1997: 190.

Material examinado. 2fe $1 \mathrm{~m}$ VII-92, 2fe 1m XII-92, Olyra sp. 
Distribuição. Brasil; Colômbia; Costa Rica; Equador; El Salvador; Honduras; Índias Ocidentais Francesas; Irã; México; Nicaragua; Venezuela.

Observações. Essa espécie foi coletada sobre bambu pelo autor sênior, em Botucatu, SP (DZSJRP n ${ }^{\circ} 4131$ e 4132, novembro de 1982).

Tetranychus armipenis Flechtmann \& Baker, 1970

Tetranychus armipenis Flechtmann \& Baker, 1970: 162. Material examinado. 1f e 2m XII-92, S. cordifolia; 1f X-92, Sida sp.

Distribuição. Argentina; Brasil; Venezuela.

Tetranychus ludeni Zacher, 1913

Tetranychus ludeni Zacher, 1913: 40

Tetranychus (Epitetranychus) ludeni Zacher, 1921: 187.

Tetranychus ludeni Pritchard \& Baker, 1955: 405

Tetranychus salviae Oudemans, 1931: 230 (sinônimo, de acordo com Pritchard \& Baker, 1955: 406)

Septanychus deviatarsus McGregor, 1950: 322 (sinônimo, de acordo com Pritchard \& Baker, 1955: 406)

Material examinado. 3f VII-92, B. rufa; 8f, $5 \mathrm{~m}$ e 3n VII-90, C. pubescens; 2f e $1 \mathrm{~m}$ XII-92, Croton sp. (Euphorbiaceae); 6f, 2m e 2n III-92, 1fe 1n IV-92, Desmodium sp.; 3f, 6m e 2n IX-92, $2 \mathrm{~m}$ e $1 \mathrm{n}$ X-92, L. lilacina; 6f, $1 \mathrm{~m}$ e $1 \mathrm{n}$ VII-92, 1f IX-92, 2f e 1m X-92, 1f, 1m e 1n XII-92, Lippia sp. (Verbenaceae); 1f III-92, 2m e 1n IV-92, 4f e 1m VI-92, 6f 2m e 6n IX-92, 2f X-92, 3f e 1m XI-92, L. speciosa; 1fIV-92, 3m e 4n VI-92, Piper sp.; $1 \mathrm{f} \mathrm{e} 1 \mathrm{n}$ IV-92, S. santaremensis; $1 \mathrm{~m}$ e 2n X-92, S. cayenensis; 1f e 1m VI-92, 3F, 2 m e 4n IX-92, $T$. semitriloba; $2 \mathrm{f}, 3 \mathrm{~m}$ e $2 \mathrm{n}$ I-91, $11 \mathrm{fe} 9 \mathrm{~m}$ IV-91, 4f, $2 \mathrm{~m}$ e 2n VII92, 3f e 1m X-92, 3F, 1f e 3m IX-92, 2f e 4m XII-92, cinco plantas não identificadas.

Distribuição. África do Sul; Alemanha; Argélia; Argentina; Austrália; Brasil; Chile; China; Colômbia; Costa Rica; Equador; El Salvador; Espanha; E.U.A.; Fiji; França; Grécia; Havaí; Honduras; Ilha Madeira; Ilhas Canárias; Ilhas Cook; Índia; Iraque; Japão; Madagascar; Malaui; Maurício; Marrocos; México; Moçambique; Nicarágua; Nova Caledônia; Nova Zelândia; Paraguai; Portugal; Peru; Polinésia Francesa; Quênia; Suíça; Taiwan; Tailândia; Tasmânia; Venezuela; Zâmbia, Zimbábue.

\section{Tetranychus mexicanus (McGregor, 1950)}

Septanychus mexicanus McGregor, 1950: 323.

Tetranychus mexicanus, Pritchard \& Baker, 1955: 411.

Material examinado. 1n V-92, 1f e $1 \mathrm{~m}$ VII-92, $A$. glandulosa; 1f VII-92, Andropogon sp.; 1f VII-92, 1f, $1 \mathrm{~m} \mathrm{e}$ In IX-92, C. iguanae; 1f XI-92, L. speciosa; $2 \mathrm{f}$ e 2m VII-92, Zanthoxyllum stipitatum C.C. Huang (Rutaceae); $11 \mathrm{f} \mathrm{e} 8 \mathrm{~m}$ 26-IV-92, 1f IX-92, plantas não identificadas.

Distribuição. Argentina; Brasil; Colômbia; Costa Rica; Cuba; El Salvador; E.U.A.; Honduras; México; Nicarágua; Paraguai; Peru; Uruguai.
Diptilomiopidae Keifer, 1944

\section{Rhynacus sp.}

Material examinado. 14 exemplares IX-92, 4 exemplares X-92, L. lilacina.

\section{Eriophyidae Nalepa, 1898}

\section{Aculops sp.}

Material examinado. 3 exemplares IV-92, 13 exemplares VI-92, S. santaremensis; 13 exemplares VII-92, 10 exemplares XII-92, planta não identificada.

\section{Aceria spp.}

Material examinado. 67 exemplares IV-92, 10 exemplares VI-92, Acalypha sp.; 10 exemplares X-92, $C$. iguanae; 30 exemplares X-92, Asteraceae não identificada; 9 exemplares X-92, 112 exemplares IX-92, 6 exemplares XII92, plantas não identificadas. hospedeiros.

Observações. Cinco espécies sobre seis diferentes

\section{Acalitus sp.}

Material examinado. 2 exemplares III-92, planta não identificada.

\section{Disella sp.}

Material examinado. 73 exemplares VII-92, Piper sp.

\section{Tetra sp.}

Material examinado. 47 exemplares IX-92, 4 exemplares XII-92, Olyra sp.

\section{Acaridida}

\section{Acaridae Ewing \& Nesbitt, 1954}

\section{Caloglyphus sp.}

Material examinado. 1f VI-92, 2f e 2m VII-92, Piper sp.; 1f VII-92, S. camporum; 1f VII-92, 2f e 1m IX-92, $C$. iguanae; 1f III-92, 2f e 3m XII-92, A. glandulosa.

Winterschmidtiidae Oudemans, 1923

\section{Czenspinskia sp.}

Material examinado. 1f III-92, Lantana sp.; 3f VII92, Vigna sp. (Fabaceae); 1f IX-92, Olyra sp.; 2f III-92, $1 \mathrm{f}$ XII-92, A. glandulosa; 3f IX-92, 3f X-92, 3 plantas não identificadas.

Oulenzia sp.

Material examinado. 1f III-92, planta não identificada.

Oribatida 


\section{Ceratozetidae Jacot, 1925}

Ceratozetes sp.

Material examinado. 1 exemplar III-92, 2 exemplares IV-92, Lantana sp.; 1 exemplar III-92, Helicteres sp.; 1 exemplar VI-92, A. glandulosa; 2f VIII-92, L. speciosa.

Galumnidae Jacot, 1925

Pergalumna sp.

Material examinado. 1 exemplar XII-92, Piper sp.

Oripodidae Jacot, 1925

Oripoda sp.

Material examinado. 1 exemplar VIII-92, L. speciosa.

Scheloribatidae Grandjean, 1933

\section{Hemileius sp.}

Material examinado. 1 exemplar III-92, 2 exemplares IV-92, 1 exemplar VIII-92, Lantana sp.; 1 exemplar III-92, 1 exemplar VI-92, L. speciosa; 1 exemplar III-92, Piper sp.; 1 exemplar X-92, B. rufa.

\section{Discussão}

Dez das 83 espécies registradas foram determinadas como novas, devendo ser descritas oportunamente. Dentre as plantas hospedeiras identificadas, aquelas que abrigaram as maiores riquezas de espécies foram Piper sp. (21), Luhea speciosa (17), Alchornea glandulosa (16), Bauhinia ruffa (14) e Olyra sp. (12). Entre os Tetranychidae, uma das mais importantes famílias de ácaros fitófagos, a espécie mais comum foi Tetranychus ludeni, encontrada sobre 15 diferentes hospedeiros. Entre os Phytoseiidae, a mais importante família de ácaros predadores em plantas, a espécie mais comum foi Iphisiodes zuluagai, encontrada sobre 14 espécies vegetais.

Este é o primeiro inventário de acarofauna realizado em área de reserva natural, com freqüência mensal de amostragem de todos os grupos taxonômicos de ácaros plantícolas.

\section{Referências Bibliográficas}

ARANDAC, B. R. \& FLECHTMANN, C.H.W. 1969. Ácaros do gênero Lorryia no Brasil e Paraguay. Resumos da II Reunião Anual Soc. brasil. Entomol., Recife: 41-42.

ATHIAS-HENRIOT, C. 1957. Phytoseiidae et Aceosejidae (Acarina, Gamasina) d'Algerie. I Genres Blattisocius Keegan, Iphiseius Berlese, Amblyseius Berlese, Phytoseius Ribaga, Phytoseiulus Evans. Bull. Soc. Hist. Nat. Afrique Nord 48:319-352.
ATTIAH, H. H. 1970. New tarsonemid mites associated with citrus in Florida (Acarina: Tarsonemidae). Florida Entomol. 53: 179-201.

BAKER, E.W. 1949a. A review of the mites of the family Cheyletidae in the United States National Museum. Proc. U.S. Natl. Mus. 99 (3238): 267-320.

BAKER, E.W. 1949b. The genus Brevipalpus (Acarina:Pseudoleptidae). Am. Midl. Nat., 42 (2): 350-402.

BAKER, E.W. 1968. The genus Lorryia. Ann. Entomol. Soc. Amer. 61 (4): 986-1008.

BAKER, E.W. 1970. The genus Tydeus : Subgenera and species groups with descriptions of new species (Acarina:Tydeidae). Ann. Entomol. Soc. Amer. 63 (1); 163-177.

BAKER, E.W. \& PRITCHARD, E.A. 1960. The tetranychoid mites of Africa. Hilgardia 29 (11): 455-574.

BAKER, E.W. \& PRITCHARD, E.A. 1962. Arañas rojas de America Central. Revta. Soc. mex. Hist. nat. 23: 309-340.

BAKER, E.W. \& TUTTLE, D.M. 1987. The false spider mites of Mexico (Tenuipalpidae:Acari). Tech. Bull. U.S. Dept. Agric. $\mathrm{n}^{\circ} 1706,237 \mathrm{p}$.

BAKER, E.W., TUTTLE, D.M. \& ABBATIELLO, M.J. 1975. The false spider mites of northwestern and north central Mexico (Acarina:Tenuipalpidae). Smith. Contrib. Zool. 194: 1-23.

BAKER, E.W. \& WHARTON, G.W. 1952. An introduction to Acarology. The MacMillan Company, New York.

BANKS, N. 1904. Class III, Arachnida, Order I, Acarina, four new species of injurious mites. Jour. N.Y. Entomol. Soc. 12:53-56.

BANKS, N. 1905. Descriptions of some new mites. Proc. Entomol. Soc. Wash., 7:133-142.

BANKS, N. 1912. New american mites. Proc. Entomol. Soc. Wash. 14: 96-98.

BANKS, N. 1915. The Acarina or mites. A review of the group for the use of economic entomologists. U.S. Dept. Agric. Report 108: 1-153.

BLANCHARD, E. 1940. Tres acaros daninos para los cultivos argentinos. Rev. Fac. Agron. La Plata (ter epoca) 24: 11-18

BOLLAND , H.R., GUTIERREZ, J. \& FLECHTMANN, C.H.W. 1998. World catalogue of the spider mite family (Acari: Tetranychidae). Brill, Leiden.

CHANT, D.A. 1959. Phytoseiid mites (Acarina:Phytoseiidae). Part I. Bionomics of seven species in southeastern England. Part II. A taxonomic review of the family Phytoseiidae, with descriptions of thirty-eight new species. Canad. Entomol. 12 (suppl.): 1-166.

CHANT, D.A. \& BAKER, E.W. 1965. The Phytoseiidae (Acarina) of Central America. Mem. Entomol. Soc. Canada 41: $1-56$. 
CHANT, D. A. \& YOSHIDA-SHAUL, E. 1984. A world review of the occidentalis species group in the genus Typhlodromus Scheuten (Acarina: Phytoseiidae). Canad. Jour. Zool. 62: 1860-1871.

CHIAVEGATO, L.G. 1980. Ácaros da cultura dos citros. In: Rodriguez, O. \& Viégas, F.C.P. (Coord.) Citricultura brasileira. Fundação Cargill, Campinas, SP. p. 469-501.

CROMROY, H.L. 1958. A preliminary survey of the plant mites of Puerto Rico. Jour. Agric. Univ. Puerto Rico 42 (2): 39-144.

COOREMAN, J. 1958. Notes et observations sur les Acariens. VII- Photia graeca n.sp. (Acaridia, Canestriniidae) et Lorryia formosa n. sp. (Stomatostigmata, Tydeydae). Bull. Inst. Roy. Sci. Nat. Belgique 34 (8): 1-10.

CUNLIFFE, F. \& BAKER, E.W. 1953. A guide to the predatory phytoseiid mites of the United States. Pinellas Biol.Lab., Inc. Pub. $n^{\circ} 1$.

DAUD, R.D. \& FERES, R.J.F. 2004. Diversidade e Flutuação Populacional de Ácaros (Acari) em Mabea fistulifera Mart. (Euphorbiaceae) de dois fragmentos de Mata Estacional Semidecídua em São José do Rio Preto, São Paulo. Neotrop. Entomol. (no prelo).

DE LEON, D. 1956. Some mites from lychee. Description of two genera and five new species of Tarsonemidae. Florida Entomol. 39 (4): 163-174.

DE LEON, D. 1957. Two new Eotetranychus and a new Oligonychus from southern Florida (Acarina: Tetranychidae). Florida Entomol. 40 (3):111-113.

DE LEON, D. 1958. Four new Typhlodromus from southern Florida (Acarina: Phytoseiidae). Florida Entomol. 41: 73-76.

DE LEON, D. 1959. A new genus and three new species of Phytoseiid mite from Mexico with collection records on Phytoseius plumifer (C. \& F.) and P. macropilis (Banks). Entomol. News 70: 147-152.

DE LEON, D. 1961a. The genus Brevipalpus in Mexico. Part II (Acarina:Tenuipalpidae). Florida Entomol. 44 (1): 41-52.

DE LEON, D. 1961b. Eight New Amblyseius from Mexico with Collection Notes on Two Other Species (Acarina: Phytoseiidae). Florida Entomol. 44(2): 85-91.

DE LEON, D. 1962 The cervices of some phytoseiid type specimens (Acarina: Phytoseiidae). Acarologia. 44: 174-176

DE LEON, D. 1965. Phytoseiid mites from Puerto Rico with descriptions of new species (Acarina: Mesostigmata). Florida Entomol. 48 (2):121-131.

DE LEON, D. 1966. Phytoseiidae of British Guyana with keys to species (Acarina: Mesostigmata). In GEIJSKES, D.C. \& HUMMELINCK, P.W. [eds]. Studies the Fauna of Suriname and other Guyanas, 8: 81-102.
DE LEON, D. 1967. Some mites of the Caribbean Area. Part I. Acarina on plants in Trinidad, West Indies. Allen Press Inc., Lawrence, Kansas.

DENMARK, H. A. 1966. Revision of the genus Phytoseius Ribaga, 1904 (Acarina: Phytoseiidae). Florida Dept. Agric. Bull. 6: 1-105.

DENMARK, H. A. 1982. Revision of Galendromus Muma, 1961 (Acarina: Phytoseiidae). ). Internat. J. Acarol. 8 (3): 133-167.

DENMARK, H.A. \& MUMA, M.H. 1970. Some phytoseiid mites of Paraguay (Phytoseiidae: Acarina). Florida Entomol. 53 (4): 219-227.

DENMARK, H.A. \& MUMA, M.H.1972. Some Phytoseiidae of the Colombia (Acarina : Phytoseiidae). Florida Entomol. 55: 19-29.

DENMARK, H.A. \& MUMA, M.H. 1973. Phytoseiid mites of Brazil (Acarina: Phytoseiidae). Revta. bras. Biol. 33: 235-276.

DENMARK, H.A. \& MUMA, M.H. 1975. The Phytoseiidae (Acari: Mesostigmata) of Puerto Rico. Jour. Agric. Univ. Puerto Rico 59 (4): 279-304.

DENMARK, H.A. \& MUMA, M.H. 1989. A revision of the genus Amblyseius Berlese, 1914 (Acari: Phytoseiidae). Occ. Pap. Florida St. Coll. Arthropods 4: 1-149.

DENMARK, H.A. \& SCHICHA, E. 1983. Revision of the genus Phytoseius Evans (Acarina: Phytoseiidae). Internat. J. Acarol. 9 (1): 27-35.

DONNADIEU, A.L. 1875. Recherches pour servir à l' histoire des Tétranyques. Ann. Soc. Linn. 22: 29-136.

EHARA, S. 1966. Some mites associated with plants on the State of São Paulo, Brazil, with a list of plant mite of South America. Jap. Jour. Zool. 15 (2): 129-149.

EHARA, S. 1993. Two new species of the genus Agistemus Summers from Malayasia (Acari, Stigmaeidae). Jour. Acarol. Soc. Jpn 2 (2): 79-82.

EVANS, G.O. 1952. On a new predatory mite of economic importance. Bull. Entomol. Res. 43: 397-401.

EWING, H. E. 1939. A revision of the mites of the subfamily Tarsoneminae of North America, the West Indies and Hawaiian Islands. United States Department of Agriculture, Technical Bulletin (653): 1-63.

FAIN, A., BOCHKOV, A.V. \& CORPUZ-RARUS, L.A. 2002. A revision of the Hemicheyletia generic group (Acari: Cheyletidae). Bull. Inst. Roy. Sci. Nat. 72: 27-66.

FARIAS, A.R., FLECHTMANN, C.H.W., MORAES, G.J. de \& McMURTRY, J. A. 1981. Predadores do ácaro verde da mandioca, no nordeste do Brasil. Pesqu. Agropec. bras. 16(3): 313-317.

FERES, R.J.F. 2000. Levantamento e observações naturalísticas da acarofauna (Acari, Arachnida) de seringueiras cultivadas (Hevea spp., Euphorbiaceae) no Brasil. Revta. bras. Zool. 17 (1): 157-173. 
FERES, R.J.F. \& FLECHTMANN, C.H.W. 1995. Mites (Acari) associated with bamboo (Bambusa sp., Poaceae) in a wood area from northwestern São Paulo State, Brazil. Revta. bras. Zool. 12 (3): 533-546.

FERES, R.J.F. \& FLECHTMANN, C.H.W. 2000. Four new Neotetranychus Trägardh (Acari, Tetranychidae) from São Paulo State, Brazil. Acarologia 41 (1-2): 215-226.

FERES, R.J.F. \& MORAES, G.J. de. 1998. Phytoseiid mites (Acari: Phytoseiidae) from wood areas in the State of São Paulo, Brazil. Syst. Appl. Acarol. 3: 125-132.

FERES, R.J.F. \& NUNES, M.A. 2001. Ácaros (Acari, Arachnida) associados a euforbiáceas nativas em áreas de cultivo de seringueiras (Hevea brasiliensis Muell. Arg., Euphorbiaceae) na região noroeste do Estado de São Paulo, Brasil. Revta. bras. Zool. 18 (4): 1253-1264.

FERLA, N.J. \& MORAES, G.J. de. 2002. Ácaros predadores (Acari) em plantas nativas e cultivadas do Estado do Rio Grande do Sul, Brasil. Rev. Bras. Zool. 19: 1011-1031.

FLECHTMANN, C.H.W. 1973. Lorryia formosa Cooremann, 1958 - Um ácaro dos citros pouco conhecido no Brasil. Ciênc. Cult. 25 (12): 1179-1181.

FLECHTMANN, C.H.W. 1975. Elementos de Acarologia. Livraria Nobel S.A., São Paulo.

FLECHTMANN, C.H.W. 1976a. Preliminary report on the false spider mites (Acari: Tenuipalpidae) from Brazil and Paraguai. Proc. Entomol. Soc. Wash. 78 (1): 58-64.

FLECHTMANN, C.H.W. 1976b. A report on the Tetranychidae (Acari) of Brazil: an emmendation. Revta. brasi. Entomol. 20 (2): 115-116.

FLECHTMANN, C.H.W. 1996. Eotetranychus tremae De Leon: Additional descriptions and illustrations (Acari, Tetranychidae). Revta bras. Zool. 13 (1): 211-214.

FLECHTMANN, C.H.W. 2004. Two new plant feeding mites from Brachiaria ruziziensis in citrus groves in São Paulo, Brazil and new distribution records of other plant mites in Brazil. Zootaxa 708:1-14

FLECHTMANN, C.H.W. \&ARLEU, R.J. 1984. Oligonychus coffeae (Nietner, 1861), um ácaro tetraniquídeo da seringueira (Hevea brasiliensis) novo para o Brasil e observações sobre outros ácaros desta planta. Ecossistema 9: 123-125.

FLECHTMANN, C.H.W. \& BAKER, E.W. 1970. A preliminary report on the Tetranychidae (Acarina) of Brazil. Ann. Entomol. Soc. Amer. 63 (1):156-163.

FLECHTMANN, C.H.W.\& BAKER, E.W. 1975. A report on the Tetranychidae (Acari) of Brazil. Revta. brasil. Entomol. 19(3): 111-122

FLECHTMANN, C.H.W. \& SANTANA, D.L.Q. 1997. A preliminary note on mites on corn in Brazil with redescriptions of Catarhinus tricholaenae and Oligonychus zeae (Acari: Diptilomiopidae, Tetranychidae). Syst. Appl. Acarol. 2:189-194.
GARMAN, P. 1958. New species belonging to the genera Amblyseius and Amblyseiopsis with keys to Amblyseius, Amblyseiopsis and Phytoseiulus. Ann. Entomol. Soc. Amer. 51: 69-79.

GEIJSKES, D.C. 1939. Beitraege zur Kenntnis der europeaischen Spinnmilben (Acari, Tetranychidae), mit besonderer Beruecksichtigung der niederlaendischen Arten. Meded. Landbouwhoogesch. Wagening. 42 (4): $1-68$.

GONDIM JR. M.G.C. \& MORAES, G. J. de. 2001. Phytoseiid mites (Acari: Phytoseiidae) associated with palm trees (Arecaceae) in Brazil. Syst. Appl. Acarol. 6: 65-94.

GONZALEZ, R.H. 1975. Revision of the Brevipalpus phoenicis "complex", with descriptions of new species from Chile and Thailand (Acarina, Tenuipalpidae). Acarologia 17 (1): 82-91.

HIRST, S. 1926. Descriptions of new mites including four new species of "red spider". Proc. Zool. Soc. London 825-841.

JEPPSON, L.R.; KEIFER, H.H. \& BAKER, E.W. 1975. Mites injurious to economic plants. University of California Press, Berkeley.

KALISZEWSKI, M. 1993. Key to Paleartic females of the genus Tarsonemus. Wydawnictwo Naukowe Uniwersytet Im. Adama Mickiewica w Poznaniu, Poznan, Seria Zoologia 14: 204 pp.

KENNETT, C.E. 1958. Some predacious mites of the subfamilies Phytoseiinae and Aceosejinae (Acarina: Phytoseiidae, Aceosejidae) from central California with descriptions of new species. Ann. Entomol. Soc. Amer. 51:471-479.

KREITER, S. \& MORAES, G.J. de. 1997. Phytoseiid mites (Acari: Phytoseiidae) from Guadeloupe and Martinique. Florida Entomol. 80 (3): 376-382.

LIN, J.Z. \& ZHANG, Z.Q. 2002. Tarsonemidae of the World (Acari:Prostigmata): Key to Genera, Geografical Distribution, Systematic Catalogue and Annotated Bibliography. Systematic and Applied Acarology Society, London.

LINDQUIST, E. E. 1978. On the synonymy of Tarsonemus waitei Banks, T. setifer Ewing, and T. bakeri Ewing, with redescription of species (Acari: Tarsonemidae). Canadian Entomol. 110(10): 1023-1048.

LINDQUIST, E. E. 1986. The word genera of Tarsonemidae (Acari: Heterostigmata): A morphological, phylogentic, and systematic revision, with a reclassification of family-group taxa in the Heterostigamata. Mem. Entomol. Soc. Canada (136): 1-517.

LIVSHITS, I.Z. \& SALINAS-CROCHE,A. 1968. Preliminares acerca de los acaros "Tetranicos" de Cuba. Cent. Natl. Fitosanid. $156 \mathrm{p}$. 
McGREGOR, E.A. 1914. Four new tetranychids. Ann. Entomol. Soc. Amer. 7:354-364.

McGREGOR, E.A. 1950. Mites of the family Tetranychidae. Am. Midl. Nat. 44 (2):257-420.

McGREGOR, E.A. 1955. Notes on spider mites (Tetranychidae) of Ecuador. Rev. Ecuat. Entomol. Parasitol. 2 (3-4): 365-377.

McMURTRY, J.A. 1983. Phytoseiidae mites from Guatemala, with descriptions of two new species and redefinitions of the genera Euseius, Typhloseiopsis, and Typhlodromus occidentalis species group (Acari: Mesostigmata). Internat. J. Acarol. 25 (4): 249-272.

McMURTRY, J.A., HUFFAKER, C.B. \& VAN de VRIE, M. 1970. Ecology of tetranychid mites and their natural enemies: a review. 1. Tetranychid enemies: Their biological characters and the impact of spray practices. Hilgardia 40 (11):331-390.

McMURTRY, J.A. \& MORAES, G.J. de. 1989. Some phytoseiid mites from Peru with descriptions of four new species (Acari: Phytoseiidae). Internat. J. Acarol. 15 (3): 179-188.

MEYER, M.K.P.S. 1974. A revision of the Tetranychidae of Africa (Acari) with a key to the genera of the world. Entomology Mem. Dep. agric. tech. Serv. Repub. S. Afr. $\mathrm{n}^{\circ} 36,291 \mathrm{p}$.

MEYER, M.K.P.S. 1979. The Tenuipalpidae (Acari) of Africa with keys to the world fauna. Entomology Mem. Dep. agric. tech. Serv. Repub. S. Afr. ${ }^{\circ} 50,135$ p.

MEYER, M.K.P.S. 1987. African Tetranychidae (Acari: Prostigmata), with reference to the world genera. Entomology Mem. Dep. Agric. Wat. Supply Repub. S. Afr. $n^{\circ}$ $69,175 \mathrm{p}$.

MEYER, M.K.P.S. \& RODRIGUES, M. da C. 1965. Acari associated with cotton in Southern Africa (with reference to other plants). Garcia de Orta 13 (2): 195-226.

MORAES, G.J. de, DENMARK, H.A. \& GUERRERO, J.M. 1982. Phytoseiid mites of Colombia (Acarina: Phytoseiidae). Internat. J. Acarol. 8 (1): 15-22.

MORAES, G.J de, KREITER, S. \& LOFEGO, A.C. 1999 (2000). Plant mites (Acari) of the French Antilles. 3. Phytoseiidae (Gamasida). Acarologia 40 (3): 237-264.

MORAES, G.J. de \& McMURTRY, J.A. 1983. Phytoseiid mites (Acarina) of northeastern Brazil with descriptions of four new species. Internat. J. Acarol. 9 (3): 131-148.

MORAES, G.J. de, McMURTRY, J.A. \& DENMARK, H.A. 1986. A catalog of the mite family Phytoseiidade. References to taxonomy, synonymy, distribution and habitat. EMBRAPA-DDT, Brasília.

MORAES, G.J. de, McMURTRY, J.A., DENMARK, H.A. \& CAMPOS, C.B. 2004. A revised catalog of the mite family Phytoseiidade (Zootaxa 434). Magnolia Press, Auckland.
MORAES, G.J. de, \& MESA, N.C. 1988. Mites of the family Phytoseiidae (Acari) in Colombia, with descriptions of three new species. Internat. J. Acarol. 14: 71-88.

MORAES, G.J. de, MESA, N.C. \& BRAUN, A. 1991. Some phytoseiid mites of Latin America (Acari: Phytoseiidae). Internat. J. Acarol. 17(2): 117-139.

MORAES, G.J. de \& OLIVEIRA, J.V. de. 1982. Phytoseiid mites of coastal Pernambuco in northeastern Brazil. Acarologia 23 (4): 315-318.

MUMA, M.H. 1955. Phytoseiidae (Acarina) associated with citrus in Florida. Ann. Entomol. Soc. Amer. 48: 262-272.

MUMA, M.H. 1961. Subfamilies, genera, and species of Phytoseiidae (Acarina: Mesostigmata). Bull. Florida St. Mus. Biol. Sci. 5 (7): 267-302.

MUMA, M.H. 1963. The genus Galendromus Muma, 1961 (Acarina: Phytoseiidae). Florida Entomol. 1(Suppl.):15-41.

MUMA, M.H. 1964. Cheyletidae (Acarina:Trombidiformes) associated with citrus in Florida. Florida Entomol. 47 (4): 239-253.

MUMA, M.H., DENMARK, H.A. \& DE LEON, D. 1970. Phytoseiidade of the Florida. Arthropods of Florida and neighboring land areas, 6. Florida Department of Agriculture and Consume Services, Division of Plant Industry, Gainesville.

OUDEMANS, A.C. 1931. Acarologische Aanteekeningen CVII. Entomol. Ber. 8(178): 221-236.

PRITCHARD, A.E. \& BAKER, E.W. (1951) 1952. The false spider mites of California (Acarina: Phytoptipalpidae). Univ. Calif. Publ. Entomol. 9(1): 1-94.

PRITCHARD, A.E. \& BAKER, E.W. 1955. A revision of the spider mite family Tetranychidae. Pacific Coast Entomological Society, Memoirs series vol.2. San Francisco.

PRITCHARD, A.E. \& BAKER, E.W. 1958. The false spider mites (Acarina: Tenuipalpidae). Univ. Calif. Publ. Entomol. $14(3): 175-274$.

RODRIGUES, M. da C. 1968. Acarina de Moçambique Catálogo das espécies relacionadas com a agricultura. Agron. moçamb. 2 (4): 215-256.

SAYED, M.T. 1946. Description of Tenuipalpus granati nov. spec. and Brevipalpus pyri nov. spec. Bull. Soc. Fouad $1^{\text {er }}$ Entomologie 30: 99-104.

SMILEY, R. L. 1969. Further studies on the Tarsonemidae, II (Acarina). Proc. Entomol. Soc. Wash. 71 (2): 218-229.

SMILEY, R. L. 1972. A review of the genus Daidalotarsonemus De Leon (Acarina: Tarsonemidae). Proc. Entomol. Soc. Wash. 74: 89-94.

SMILEY, R.L. 1975. A generic revision of the mites of the family Cunaxidae (Acarina). Ann. Entomol. Soc. Amer. 68(2): 227- 244. 
SUMMERS, F.M. \& PRICE, D.W. 1970. Revision of the family Cheyletidae. Univ. Calif. Publ. Entomol. 61: 1-153.

SUSKI, Z. W. 1965. Tarsonemid mites on apple trees in Poland. II. Tarsonemus bilobatus n. sp. (Acarina, Tasonemidae). Bull. Acad. Polonaise Sci. 13 (9): 534-544.

SUSKI, Z. W. 1967. Tarsonemid mites on apple trees in Poland. IX. Tarsonemus pauperoseatus n. sp. (Acarina, Heterostigmata). Bull. Acad. Polonaise Sci. 15(5): 267-272.

TUTTLE, D.M. \& BAKER, E.W. 1968. Spider mites of southwestern United States and a revision of the family Tetranychidae. The University of Arizona Press, Tucson, Arizona.

TUTTLE, D.M., BAKER, E.W.\&ABBATIELLO, M. 1974. Spider mites from northwestern and north central Mexico (Acarina: Tetranychidae). Smithson. Contrib. Zool. 171: 1-17.

TUTTLE, D.M., BAKER E.W. \& ABBATIELLO, M. 1976. Spider mites of Mexico (Acari: Tetranychidae). Internat. J.Acarol. 2(2): 1-102.

VOLGIN, V.I. 1955. In Paulovskii, E.N. Acarina of rodents of the USSR fauna. Akad. Nauk SSSR, Zool. Inst., Opredelitelipo faune SSSR 59: 1-459.

VOLGIN, V.I. 1969. Acarina of the family Cheyletidae of the World. Akad. Nauk. SSSR Zool. Inst. Opredelitelipo faune SSSR 59:1-495.

WAINSTEIN, B.A. 1960. Tetranychoid mites of Kazakhstan (with revision of the family). Kazakh. Akad. Sel'sk Nauk Nauch. Issled Inst. Zash. Rast. 5: 1-276.

WAINSTEIN, B.A. 1971. Mononychellus, a new name for Mononychus (Acariformes, Tetranychidae). Zool. Zhur. 50(4):589.

WILSON, E.O. 1997. A situação atual da diversidade biológica, pp. 3-24. In: Wilson, E.O. (org.), Biodiversidade. Editora Nova Fronteira, Rio de Janeiro

WOOD, T.G. 1967. New Zealand mites of the family Stigmaeidae (Acari, Prostigmata). Trans. Roy. Soc. New Zeland 9(9): 93-139.

WOOLLEY, T.A. 1988. Acarology: mites and human welfare. Library of Congress cataloging in Publication, Fort Collins, Colorado.

ZACARIAS, M.S. \& MORAES, G.J. de. 2001. Phytoseiid mites (Acari) associated with rubber trees and other Euphorbiaceous plants in southeastern Brazil. Neotrop. Entomol. 30: 579-586.

ZACHER, F. 1913. Untersuchungen über Spinnmilben. Mitt. K. biol. Anst. u. Forstw. 14:37-41.

ZACHER, F. 1921. Neue und wenig bekannte Spinnmilben. Z. Angew. Entomol. 7: 181-187.

ZULUAGA, C.\&SALDARRIAGA, V. 1970. Reconocimiento, identificacion y algunas observaciones sobre dinamica de poblaciones de acaros, en citricos del valle del Cauca. Acta Agron. 20 (3/4): 114-141.
Título: Ácaros Plantícolas (Acari) da "Estação Ecológica do Noroeste Paulista”, Estado de São Paulo, Brasil

Autores: Reinaldo José Fazzio Feres, Antonio Carlos Lofego \& Anibal Ramadan Oliveira

Biota Neotropica, Vol. 5 ( número 1): 2005

http://www.biotaneotropica.org.br/v5 n 1/pt/ abstract?article+BN00405012005

Recebido: 2/08/2004

Revisado: 11/11/2004

Publicado: 01/01/2005

ISSN 1676-0603 